\title{
Bias errors due to leakage effects when estimating frequency response functions
}

\author{
Andreas Josefsson ${ }^{\mathrm{a}, *}$, Kjell Ahlin ${ }^{\mathrm{a}}$ and Göran Broman ${ }^{\mathrm{a}, \mathrm{b}}$ \\ ${ }^{a}$ School of Engineering, Blekinge Institute of Technology, Karlskrona, Sweden \\ ${ }^{\mathrm{b}}$ Department of Functional Product Development, Luleå University of Technology, Luleå, Sweden
}

Received 23 September 2011

Revised 23 December 2011

\begin{abstract}
Frequency response functions are often utilized to characterize a system's dynamic response. For a wide range of engineering applications, it is desirable to determine frequency response functions for a system under stochastic excitation. In practice, the measurement data is contaminated by noise and some form of averaging is needed in order to obtain a consistent estimator. With Welch's method, the discrete Fourier transform is used and the data is segmented into smaller blocks so that averaging can be performed when estimating the spectrum. However, this segmentation introduces leakage effects. As a result, the estimated frequency response function suffers from both systematic (bias) and random errors due to leakage. In this paper the bias error in the $\mathrm{H}_{1}$ and $\mathrm{H}_{2}$-estimate is studied and a new method is proposed to derive an approximate expression for the relative bias error at the resonance frequency with different window functions. The method is based on using a sum of real exponentials to describe the window's deterministic autocorrelation function. Simple expressions are derived for a rectangular window and a Hanning window. The theoretical expressions are verified with numerical simulations and a very good agreement is found between the results from the proposed bias expressions and the empirical results.
\end{abstract}

Keywords: Frequency response functions, bias error, leakage effects, Welch's method

\section{Introduction}

Estimation of Frequency Response Functions (FRFs) from experimental data is commonly carried out as a first step in the system identification process. The FRFs provide a condensed overview of the system's dynamic response and often form the basis for selection of a more sophisticated, parametric, identification algorithm. This is the case in experimental modal analysis where many parametric curve-fitting routines identify the model order and corresponding modal parameters from measured FRFs. Hence, getting accurate FRF-estimates from experimental data is critical for the subsequent analysis. Being able to understand, predict and, to some extent, compensate for possible errors in the FRFs is therefore valuable.

This paper is concerned with the frequency-domain approach known as Welch's method, which uses the discrete Fourier transform and achieve averaging by segmenting the data as initially proposed in [1]. This is the most common estimator implemented in signal analysis software due to its speed and low memory requirement. However, it is also well known that this method introduces leakage errors in the spectral densities since only a finite measurement time is studied. This windowing of the data introduces spectral smoothing and ripple in the signals' spectral density. As a result, the FRF estimator is affected by leakage errors since it is calculated from the spectral densities.

In practice, the measurement data is also contaminated by measurement noise. Systematic (bias) errors due to measurement noise can normally be avoided depending on how the FRF-estimator is formulated $\left(H_{1}, H_{2}, H_{c}\right.$, etc.).

\footnotetext{
* Corresponding author: Andreas Josefsson, School of Engineering, Blekinge Institute of Technology, SE-371 79 Karlskrona, Sweden. Tel.: +46 730 336306; E-mail: andreas.josefsson@bth.se.
} 
But the estimator will still be affected by a random error due to measurement noise and thus it is necessary to segment the data and perform averaging over several blocks of data to obtain a consistent estimate. On the other hand, this segmentation of the data makes the leakage effects more severe. As a consequence, for a given input and output signal, a compromise needs to be made between the number of segments and the length on each segment when estimating the FRF.

A comparison of transfer function estimators was shown in [2-4] where the bias and variance due to leakage were studied for the $H_{1}$-estimate and compared with the raw quotient between the Fourier transform of the output and the input. More recently, an extensive study on the bias and variance of the $H_{1}$-estimate was given in [5-7] where different window functions and overlapping strategies were investigated. In [8,9] an approximate expression for the bias error at the resonance frequency was derived for a single-degree-of-freedom system with white noise input. The bias expression was found by assuming that the estimation is accomplished with an ideal rectangular spectral window, which is an approximation that is not often appropriate in practice.

The aim of this paper is to derive a bias expression which shows better agreement with empirical results. The study is carried out for the $H_{1}$-estimate, the $H_{2}$-estimate and the coherence function assuming white noise excitation. Specifically, an approximation based on using a sum of real exponentials to describe the window's deterministic autocorrelation function is used to derive an expression for the relative bias error at the resonance frequency. Finally, the accuracy of the proposed expressions is examined with numerical simulations.

\section{System identification in the frequency domain}

The problem studied is summarized below. For a single-input-single-output case an input signal, $x[n]$, and an output signal, $y[n]$, both of length $L$ samples, and both normally distributed and stationary random sequences, are given as

$$
\begin{aligned}
& x[n]=\left\{x_{0}, x_{1}, \ldots, x_{L-1}\right\} \\
& y[n]=\left\{y_{0}, y_{1}, \ldots, y_{L-1}\right\}
\end{aligned}
$$

It is assumed that the output signal is the input signal convolved with a linear, time-invariant and causal system impulse response, $h[n]$, with some added measurement noise, $u[n]$. In the $H_{1}$-case, it is assumed that the output signal cannot be measured without noise, Eq. (3), while in the $\mathrm{H}_{2}$-case it is assumed that the input signal cannot be measured without noise, Eq. (4).

$$
\begin{aligned}
& y[n]-u[n]=\sum_{k=0}^{\infty} h[k] \cdot x[n-k] \\
& y[n]=\sum_{k=0}^{\infty} h[k] \cdot(x[n-k]-u[n-k])
\end{aligned}
$$

The goal is then to find the best estimate of the linear system. With Welch's method the problem is solved by dividing the given signals in $M$, possibly overlapping, segments, each with length $N$ samples. A window function, $w[n]$, is then applied to each segment and the discrete Fourier transform is calculated, for $m=\{0,1,2 \ldots M-1\}$, as

$$
\begin{aligned}
X_{m}[k] & =\sum_{n=0}^{N-1} w[n] \cdot x_{m}[n] \cdot e^{-\frac{j 2 \pi k n}{N}} \\
Y_{m}[k] & =\sum_{n=0}^{N-1} w[n] \cdot y_{m}[n] \cdot e^{-\frac{j 2 \pi k n}{N}}
\end{aligned}
$$

The spectral densities are then estimated by multiplying with the complex conjugate and averaging over $M$ blocks, as shown in Eq. (7) for the $H_{1}$-estimate or in Eq. (8) for the $H_{2}$-estimate. 


$$
\begin{aligned}
& \hat{H}_{1}[k]=\frac{\sum_{m=0}^{M-1} Y_{m} \cdot X_{m}^{*}}{\sum_{m=0}^{M-1} X_{m} \cdot X_{m}^{*}} \\
& \hat{H}_{2}[k]=\frac{\sum_{m=0}^{M-1} Y_{m} \cdot Y_{m}^{*}}{\sum_{m=0}^{M-1} X_{m} \cdot Y_{m}^{*}}
\end{aligned}
$$

Since the ratio between these two estimators can be used as a quality measure of the estimated FRF, the ordinary coherence function is defined as

$$
\hat{\gamma}_{y x}^{2}=\frac{\hat{H}_{1}}{\hat{H}_{2}}
$$

The coherence will give a value between zero and unity at each frequency, indicating how much of the measured output that can be explained with a linear model and the measured input. Possible bias error due to measurement noise can be avoided in either the $H_{1}$ or the $H_{2}$-case (assuming that the noise is uncorrelated with the input signal). However, the estimators - including the coherence function - still suffer from random error due to measurement noise as well as, due to the finite-length record in Eqs (5)-(6), a bias- and random-error due to the leakage.

\section{Bias errors due to leakage in the $H_{1}$ - and $H_{2}$-estimate}

The technique described above assumes that a windowed output block is identical to the system response of the corresponding windowed input block which is true only if the linear system is an ideal delta-function. In practice, transient terms from previous input blocks will always be present in a studied output block. Hence, any possible correlation with previous input blocks is neglected with Welch's method.

The bias error in the estimates due to leakage can be determined by studying the bias error in spectral densities estimates, since the FRF is calculated as the quotient between two spectral densities. Bias error in spectral densities estimates has been studied in for instance [9-11].

First, the discrete normalized (deterministic) autocorrelation function of the window, as a function of lag $l$, is defined with Eqs (10)-(11).

$$
\begin{aligned}
& k_{w w}[l]= \begin{cases}\frac{1}{N} \sum_{n=0}^{N-l-1} w[n+l] \cdot w[n] & l \geqslant 0 \\
k_{w w}[-l] & l<0\end{cases} \\
& r_{w w}[l]=\frac{1}{k_{w w}(0)} \cdot k_{w w}[l]
\end{aligned}
$$

A biased cross-correlation function of two windowed signal blocks can then be written as [10]

$$
\hat{r}_{y x}[l]=\mathbf{E}\left\{y_{m}[n] \cdot w[n] \cdot x_{m}[n-l] \cdot w[n-l]\right\}=r_{y x}[l] \cdot r_{w w}[l]
$$

where $\mathbf{E}\{$.$\} denotes the expected value. Similarly, a biased autocorrelation function of y[n]$ is given by

$$
\hat{r}_{y y}[l]=\mathbf{E}\left\{y_{m}[n] \cdot w[n] \cdot y_{m}[n-l] \cdot w[n-l]\right\}=r_{y y}[l] \cdot r_{w w}[l]
$$

If the excitation signal is assumed to be white noise with variance $\sigma_{x}^{2}$, the following relationships can be used [10, 11]

$$
\begin{aligned}
& r_{y y}[l]=\sigma_{x}^{2} \cdot k_{w w}[0] \cdot r_{h h}[l] \\
& r_{y x}[l]=\sigma_{x}^{2} \cdot k_{w w}[0] \cdot h[l]
\end{aligned}
$$

where $r_{h h}[l]$ denotes the discrete, deterministic, auto-correlation function of the impulse response. In Eqs (14) and $(15), \sigma_{x}^{2} \cdot k_{w w}[0]$ denotes the variance of the windowed excitation signal $x[n]$. Using Eqs (7) and (8) gives an expression for the biased estimates as 

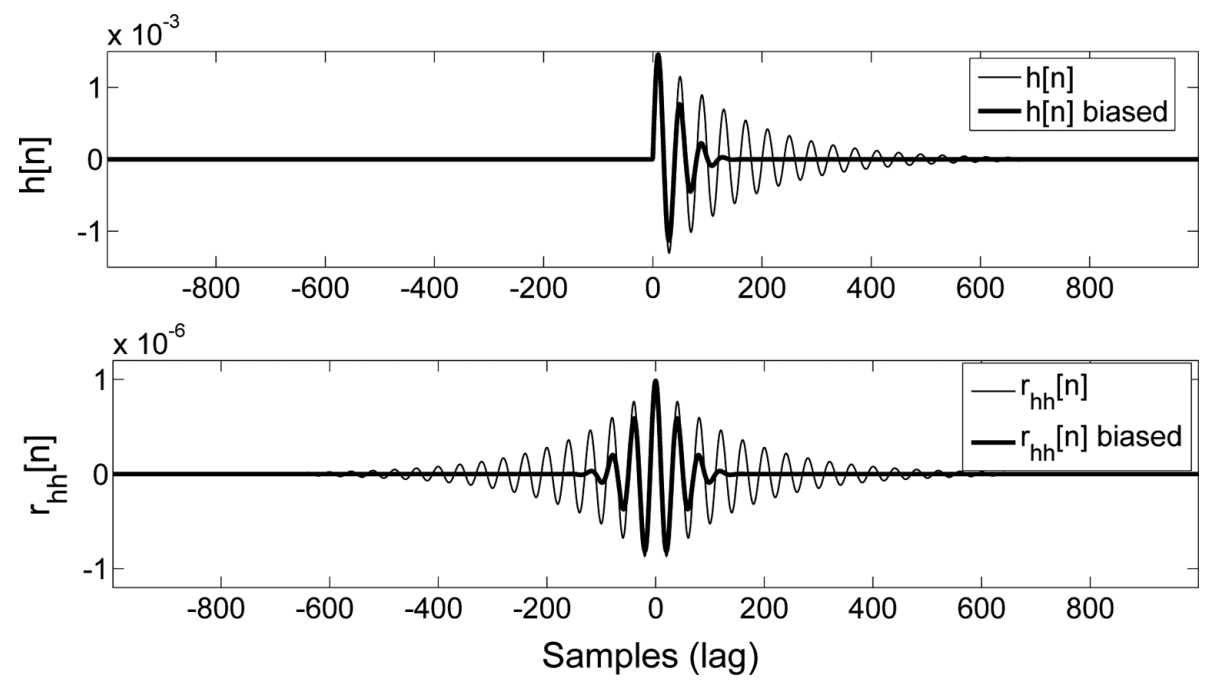

Fig. 1. The biased $H_{1}$-estimate is obtained by taking the Fourier transform of a truncated impulse response (above). For the $H_{2}$-estimate, the autocorrelation function of the impulse response is truncated (below) which gives a biased magnitude square function in frequency domain. The latter is then divided with the conjugate of the biased $\mathrm{H}_{1}$-estimate in order to obtain the $\mathrm{H}_{2}$-estimate.

$$
\begin{aligned}
\hat{H}_{1}[k] & =\sum_{n=0}^{N-1} h[n] \cdot r_{w w}[n] \cdot e^{-\frac{j 2 \pi k n}{N}} \\
\hat{H}_{2}[k] & =\frac{\sum_{n=0}^{N-1} r_{h h}[n] \cdot r_{w w}[n] \cdot e^{-\frac{j 2 \pi k n}{N}}}{\sum_{n=0}^{N-1} h[-n] \cdot r_{w w}[n] \cdot e^{-\frac{j 2 \pi k n}{N}}}
\end{aligned}
$$

Studying Eqs (16)-(17), it can be seen that the window function effectively truncates the true impulse response function. The biased $H_{1}$-estimate is obtained by taking the Fourier transform of a truncated impulse response, while the $\mathrm{H}_{2}$-estimate is obtained as a quotient between the Fourier transform of a biased autocorrelation function of the impulse response and the conjugate of the biased $H_{1}$-estimate. An example is given in Fig. 1 where the biased impulse response and the biased autocorrelation function of the impulse response are illustrated.

\section{A study of a single-degree-of-freedom system}

The relative bias error obtained at the resonance frequency for a single-degree-of-freedom (SDOF) system is considered next. In experimental modal analysis it is common to describe a general linear system with a set of modal parameters such as natural frequencies, mode shapes and modal damping. The FRFs between any measurement points can then be synthesized from the modal model. Hence, by relating the bias error to the modal parameters it is possible to obtain an estimate of the bias error in the FRFs at each modal frequency.

Studying a linear, second-order, continuous-time, system with input $x(t)$ and output $y(t)$

$$
\ddot{y}(t)+2 \zeta \omega_{0} \cdot \dot{y}(t)+\omega_{0}^{2} \cdot y(t)=d \cdot \omega_{0}^{2} \cdot x(t)
$$

where $\zeta$ denotes the relative damping, $\omega_{0}$ is the undamped resonance frequency and $d$ is a real constant. The causal impulse response to this system, in continuous time $\tau$, is given by [12]

$$
h(\tau)=\frac{d \cdot \omega_{0}}{\sqrt{1-\zeta^{2}}} \cdot e^{-\zeta \omega_{0} \tau} \cdot \sin \left(\omega_{0} \tau \sqrt{1-\zeta^{2}}\right)
$$

and, for $\zeta \leqslant 1 / \sqrt{2}$, the peak value in the FRF occurs at $\omega_{1}=\omega_{0} \sqrt{1-2 \zeta^{2}}$ and can be written as [9] 


$$
\left|H\left(\omega_{1}\right)\right|=\frac{d}{2 \zeta \sqrt{1-\zeta^{2}}}
$$

If the system is excited by a random input and Welch's method is used to calculate the FRF, a bias error will be introduced as shown in previous chapter. The difference between the true peak value at resonance frequency and the biased peak value is considered here, and a normalized bias error can be defined as shown in Eq. (21) where $\left|\hat{H}\left(\omega_{1}\right)\right|$ denotes the estimated quantity.

$$
\epsilon_{b}=\frac{\mathbf{E}\left\{\left|\hat{H}\left(\omega_{1}\right)\right|\right\}-\left|H\left(\omega_{1}\right)\right|}{\left|H\left(\omega_{1}\right)\right|}
$$

This bias error will depend on the frequency resolution, window type (e.g. rectangular, Hanning) as well as system properties (resonance frequency and relative damping). For the proceeding calculations, the input excitation is assumed to be white noise so that Eqs (16)-(17) are applicable and the relative damping is assumed to be small so that $\omega_{0} \approx \omega_{1}$ for $\zeta^{2} \approx 0$. Furthermore, it will be assumed that the continuous, deterministic, normalized, autocorrelation function of the window can be approximated as a sum of real exponentials, i.e.

$$
r_{w w}(\tau) \approx \frac{1}{A_{s}} \sum_{n=0}^{P} a_{n} \cdot e^{-k_{n}|\tau|}
$$

where $\left\{a_{n}, k_{n}\right\}$ are real-valued constants, $A_{s}=\sum_{n=0}^{P} a_{n}$ and $k_{n}>0$. As it is shown in the next section, Eq. (22) is a reasonable approximation given the shape of the windows typically used.

Starting with the $H_{1}$-estimate, Eq. (16) can be combined with Eq. (22) and the truncated impulse response can be written as

$$
h_{1}(\tau)=h(\tau) \cdot r_{w w}(\tau) \approx \frac{1}{A_{s}} \sum_{n=0}^{P} h(\tau) \cdot a_{n} \cdot e^{-k_{n}|\tau|}
$$

A multiplication with a single real exponential only changes the relative damping of the system [9]. As a result, the truncated impulse response can be seen as the sum of $P+1$ impulse responses, each with modified relative damping and each weighted with the $a_{n}$-coefficients. Using Eq. (20), and assuming that the relative damping is small so that each frequency response has a purely imaginary value at the resonance, the biased peak value can be written as

$$
\left|\hat{H}_{1}\left(\omega_{0}\right)\right| \approx \frac{1}{A_{s}} \sum_{n=0}^{P} \frac{a_{n} \cdot d}{2 \cdot \zeta_{n}}
$$

where $\zeta_{n}=\zeta+k_{n} / \omega_{0}$. By using the following relationship

$$
\sum_{n=0}^{P} \frac{a_{n}}{\zeta \omega_{0}+k_{n}}=\int_{0}^{\infty} e^{-\zeta \omega_{0} \tau} \cdot \sum_{n=0}^{P} a_{n} \cdot e^{-k_{n}|\tau|} d \tau
$$

an approximation can now be derived for the relative bias error by combining Eqs (21)-(25) which gives

$$
\epsilon_{b}^{\left|H_{1}\right|} \approx \zeta \omega_{0} \cdot \int_{0}^{\infty} e^{-\zeta \omega_{0} \tau} \cdot r_{w w}(\tau) d \tau-1
$$

Next, the relative bias error obtained at the resonance when using the $H_{2}$-estimate is examined. Studying the numerator in Eq. (17), the deterministic autocorrelation function of the impulse response can be written as [8]

$$
r_{h h}(\tau) \approx \frac{d^{2} \omega_{0}}{4 \zeta} \cdot e^{-\zeta \omega_{0}|\tau|} \cdot\left[\cos \left(\omega_{0}|\tau|\right)+\zeta \sin \left(\omega_{0}|\tau|\right)\right]
$$

when $\sqrt{1-\zeta^{2}} \approx 1$. If this function is weighted with a single real exponential, the biased magnitude square function can be written as 


$$
\begin{aligned}
\mathcal{F}\left(r_{h h} \cdot e^{-k|\tau|}\right) \approx & \left.\frac{d}{4} \mathcal{F}\left(d \omega_{0} \cdot e^{-\zeta \omega_{0}|\tau|} \cdot \sin \left(\omega_{0}|\tau|\right) \cdot e^{-k|\tau|}\right)\right) \\
& \left.+\frac{d}{4 \zeta} \mathcal{F}\left(d \omega_{0} \cdot e^{-\zeta \omega_{0}|\tau|} \cdot \cos \left(\omega_{0}|\tau|\right) \cdot e^{-k|\tau|}\right)\right)
\end{aligned}
$$

where $\mathcal{F}($.$) denotes the Fourier transform. The value at the resonance can be approximated as$

$$
\begin{aligned}
\mathcal{F}\left(r_{h h}(\tau) \cdot e^{-k|\tau|}\right)_{\omega=\omega_{0}} \approx & \frac{d}{4}\left(\frac{d}{j 2 \zeta_{0}}+\frac{d}{-j 2 \zeta_{0}}\right)+ \\
& \frac{d}{4 \zeta}\left(\frac{d\left(\zeta_{0}+j\right)}{j 2 \zeta_{0}}+\frac{d\left(\zeta_{0}-j\right)}{-j 2 \zeta_{0}}\right)=\frac{d}{2 \zeta} \cdot \frac{d}{2 \zeta_{0}}
\end{aligned}
$$

when higher-order terms, $\zeta^{2}$ and $\zeta_{0}^{2}$ are neglected. In Eq. (29), $\zeta_{0}$ denotes the modified relative damping given by $\zeta_{0}=\zeta+k / \omega_{0}$. Extending this to the general form of $r_{w w}(\tau)$, shown in Eq. (22), gives the numerator in Eq. (17) as

$$
\left|\hat{H}\left(\omega_{0}\right)\right|^{2} \approx \frac{d}{2 \zeta} \cdot \frac{1}{A_{s}} \sum_{n=0}^{P} \frac{a_{n} \cdot d}{2 \cdot \zeta_{n}}
$$

Since the denominator in Eq. (17) can be described with Eq. (24) it follows that the $\mathrm{H}_{2}$-estimate gives the true peak value as given by Eq. (20). Hence,

$$
\epsilon_{b}^{\left|H_{2}\right|} \approx 0
$$

Using Eq. (9) it then follows that the coherence value at the resonance can be approximated with

$$
\hat{\gamma}_{y x}^{2} \approx \epsilon_{b}^{\left|H_{1}\right|}+1
$$

The bias in the $H_{1}$-estimate, as given by Eq. (26), can be calculated when the window type is given. Consider, as an example, the rectangular window and the Hanning window which have deterministic autocorrelation functions given by [13]

$$
\begin{aligned}
r_{w w}^{R e c t}(\tau)=\left(1-|\tau| \cdot \Delta_{f}\right) \cdot\left(1-\theta\left(|\tau|-\frac{1}{\Delta_{f}}\right)\right) \\
r_{w w}^{\text {Hann }}(\tau)=\left(\left(1-|\tau| \cdot \Delta_{f}\right) \cdot\left(\frac{2}{3}+\frac{1}{3} \cos \left(2 \pi \Delta_{f} \cdot \tau\right)\right) \ldots\right. \\
\left.\ldots+\frac{1}{2 \pi} \sin \left(2 \pi \Delta_{f} \cdot|\tau|\right)\right) \cdot\left(1-\theta\left(|\tau|-\frac{1}{\Delta_{f}}\right)\right)
\end{aligned}
$$

where $\theta(\tau)$ denotes the step function and $\Delta_{f}$ is the frequency increment. Using Eq. (33) in Eq. (26) and carrying out the calculations gives the relative bias error for the rectangular window as

$$
\epsilon_{b}^{\left|H_{1}\right|} \approx \frac{\Delta_{f}}{\pi B_{r}}\left(e^{-\pi B_{r} / \Delta_{f}}-1\right)
$$

where $B_{r}$ is the $3 \mathrm{~dB}$-bandwidth for the system [9] defined as $B_{r}=\zeta \omega_{0} / \pi$. Similarly, combining Eq. (26) with Eq. (34) gives the relative bias error for the Hanning window as

$$
\epsilon_{b}^{\left|H_{1}\right|} \approx \frac{2 \Delta_{f}}{3}\left(\frac{1}{\pi B_{r}}+\frac{B_{r}^{3}}{\pi A^{2}}-\frac{2 B_{r}}{\pi A}\right)\left(e^{-\pi B_{r} / \Delta_{f}}-1\right)+\frac{B_{r}^{2}}{3 A}-\frac{1}{3}
$$

with $A=B_{r}^{2}+4 \Delta_{f}^{2}$. Hence, if the modal damping and resonance frequency are given or estimated, Eqs (35)-(36) can be used to approximate the bias error at the resonance frequency. 


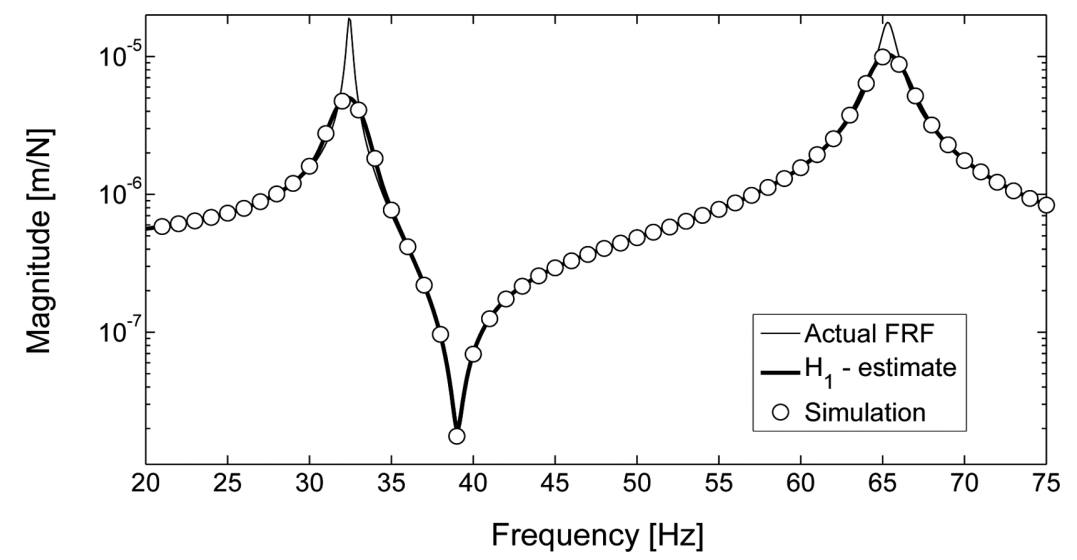

Fig. 2. A simulation on a mass-spring-damper system is performed and the result with Welch's method and the $H_{1}$-formulation is compared with the system's true FRF. After averaging, a relatively large bias error is obtained at the resonances. Finally, the simulation results are verified by comparing with the theoretical biased FRF (-) as given in Eq. (16).

\section{Numerical simulations and comparisons}

Results from numerical simulations are shown next where the bias error is demonstrated and compared with the previously derived formulas. All simulations are performed with white noise excitation and the time-response is calculated using ramp-invariant digital filters [14].

At first a discrete mass-spring-damper system with two degrees of freedom is studied with matrices given by

$$
\begin{aligned}
& {[M]=\left[\begin{array}{cc}
20 & 0 \\
0 & 25
\end{array}\right] \mathrm{kg}, \quad[C]=\left[\begin{array}{cc}
90 & -50 \\
-50 & 80
\end{array}\right] \mathrm{Ns} / \mathrm{m}} \\
& {[K]=\left[\begin{array}{cr}
3 & -1 \\
-1 & 1.5
\end{array}\right] \cdot 10^{6} \mathrm{~N} / \mathrm{m}}
\end{aligned}
$$

The simulation is performed with a sampling frequency of $2048 \mathrm{~Hz}$, and Welch's estimator is used for the analysis with 50 percent overlap and a Hanning window. No external noise is added to the simulated data, i.e. $u(n)=0$, and the high sampling frequency ensures that truncation errors when calculating the time-responses are negligible. The errors in the estimated FRFs are then dominated by a random error due to leakage and a bias error due to leakage. 16384 averages are taken in order to remove the random error and 2048 points are used for the fft-blocksize giving a frequency resolution of $\Delta_{f}=1 \mathrm{~Hz}$.

Both the $H_{1}$ - and $H_{2}$-formulations are tested in the analysis. Figure 2 shows the actual FRF for the system together with the estimated FRF when using the $H_{1}$-estimate. Similarly, Fig. 3 shows the actual FRF together with the estimated FRF when using the $\mathrm{H}_{2}$-estimate.

When comparing the actual FRF for the system with the estimated FRFs it is clear that the fft-blocksize is small in this example and relatively large bias errors are obtained. In the $H_{1}$-case, this can be seen around the resonance peaks in Fig. 2, while in the $\mathrm{H}_{2}$-case the biased FRF tend to broaden the resonance peaks and gives a larger bias error at the anti-resonance as shown in Fig. 3.

The theoretical biased FRFs can be obtained by using the true impulse response for the system and the deterministic autocorrelation function of the window as given in Eq. (11). Using Eqs (16)-(17) then produces the biased FRFs, which agrees well with the simulation results as shown in Figs 2 and 3.

Next, the bias error at the resonance frequency for a single-degree-of-freedom is considered and the formulas derived in Section 4 are compared with simulation results. In the derivation, it was assumed that the deterministic autocorrelation function of the window can be approximated with a sum of real exponentials. The validity of this assumption is demonstrated in Fig. 4 for a 256-point rectangular window and a Hanning window. Referring to Eq. (22), $100 k_{n}$-values are generated for this example and the best $a_{n}$ values are found with a least-square technique, which yields a very good approximation of the window functions as shown in Fig. 4. 


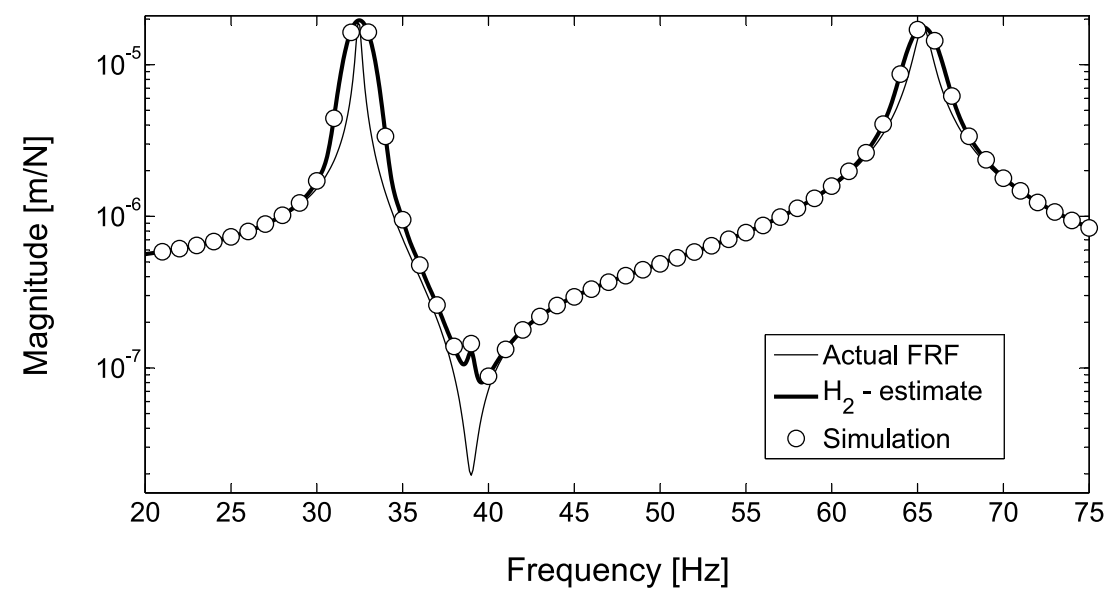

Fig. 3. Based on the same system as in Fig. 2 but the analysis is carried out with the $H_{2}$-formulation. In this case, the estimated FRF over-predicts the true FRF value before and after the resonance and gives a larger bias error at the anti-resonances. The simulation results agrees well with the theoretical biased FRF (-) as given in Eq. (17) for the $H_{2}$-estimate.
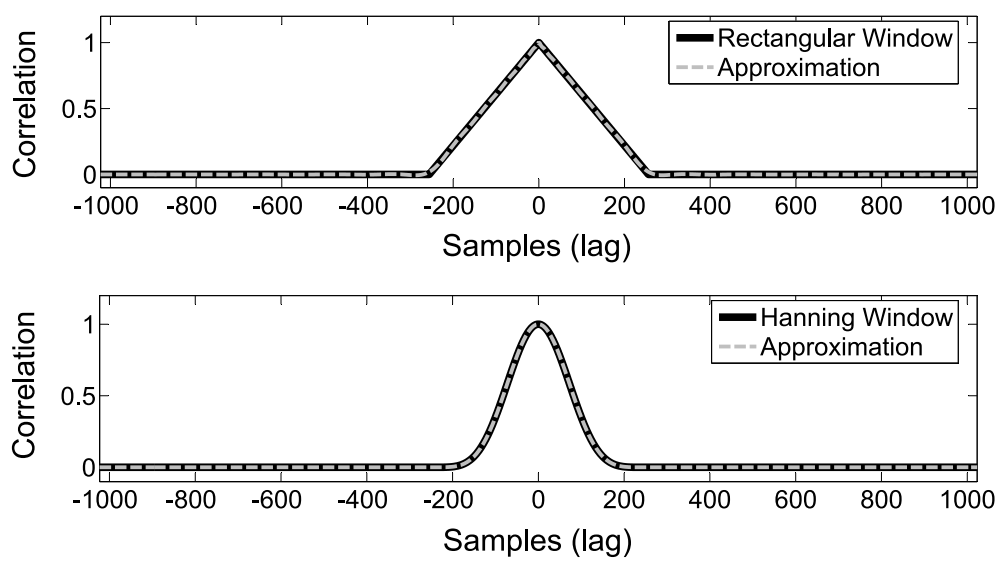

Fig. 4. The discrete deterministic autocorrelation function of a rectangular window (above) and a Hanning window (below) is shown together with a curve fit consisting of a sum of real exponentials as given in Eq. (22). 100 coefficients are used in this example.

The accuracy of the proposed bias expressions for the rectangular window and the Hanning window is demonstrated next. The time-response from a second-order system, with relative damping $\zeta=0.005$ and natural frequency $f_{0}=$ $50 \mathrm{~Hz}$, is simulated using a sampling frequency of $F s=2000 \mathrm{~Hz}$.

The theoretical FRF value at the resonance frequency is then compared with the estimated FRF value, as obtained with Welch's method, and the relative bias error, as defined in Eq. (21), is calculated. This is repeated with increasing fft-blocksizes in order to get the bias error as a function of frequency resolution. The analysis is carried out with 50 percent overlap and 100000 averages for both the rectangular window and the Hanning window.

Simulation results for the $H_{1}$-estimate agree well with results from Eqs (35)-(36) as shown in Fig. 5 and Table 1. It can be observed that the Hanning window gives a smaller bias error when the frequency increment is low but a larger bias error at a larger frequency increment. However, it should be emphasized that Fig. 5 only demonstrates the accuracy of the derived bias expressions. For a meaningful comparison between window functions it is necessary to also study the variance due to leakage noise.

Table 1 shows the simulation results for the Hanning window where the $H_{2}$-estimate and the coherence value at the resonance are included as well. The $H_{2}$-estimate is close to zero and the coherence value depends on the relative bias error in the $H_{1}$-estimate as discussed in Section 4. 
Table 1

The relative bias error at the resonance frequency obtained with the $H_{1}$ and $H_{2}$-estimate and the coherence value for the Hanning window, together with theoretical results for different frequency increments

\begin{tabular}{|c|c|c|c|c|c|c|}
\hline \multirow[b]{2}{*}{$\Delta_{f}[H z]$} & \multicolumn{2}{|c|}{ Rel. Bias Error, $H_{1}$} & \multicolumn{2}{|c|}{ Rel. Bias Error, $H_{2}$} & \multicolumn{2}{|c|}{ Coherence Value } \\
\hline & Simulation & Eq. (35) & Simulation & Eq. (31) & Simulation & Eq. (32) \\
\hline 1 & -0.612 & -0.612 & 0.002 & 0 & 0.388 & 0.389 \\
\hline $5 / 6$ & -0.557 & -0.558 & -0.001 & 0 & 0.444 & 0.441 \\
\hline $2 / 3$ & -0.491 & -0.489 & -0.001 & 0 & 0.510 & 0.511 \\
\hline $1 / 2$ & -0.397 & -0.397 & -0.001 & 0 & 0.604 & 0.603 \\
\hline $1 / 3$ & -0.271 & -0.270 & -0.003 & 0 & 0.731 & 0.729 \\
\hline $1 / 4$ & -0.194 & -0.193 & 0.002 & 0 & 0.805 & 0.807 \\
\hline $1 / 6$ & -0.109 & -0.109 & -0.000 & 0 & 0.891 & 0.890 \\
\hline $1 / 14$ & -0.025 & -0.025 & -0.000 & 0 & 0.975 & 0.975 \\
\hline
\end{tabular}

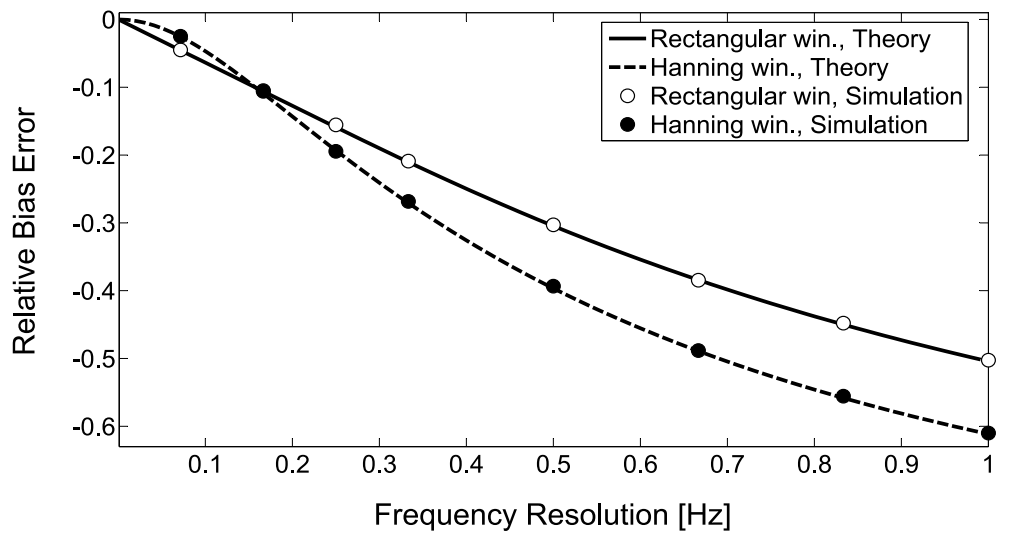

Fig. 5. An FRF is calculated from simulated time-responses for a single-degree-of-freedom system. The result is compared with the true FRF and the relative bias error at the resonance is determined. This is done for both the rectangular window and the Hanning window using the $H_{1}$-formulation. Numerical results, which agree well with the proposed bias expressions, are shown with increasing frequency increment.

\section{Discussion}

Calculation of frequency-response-functions (FRFs) from finite data sequences often requires a segmentation of the data (Welch's method) in order to obtain a consistent estimator when contaminating measurement noise is present. This segmentation can in turn introduce severe stochastic errors due the leakage noise. In practice, it is necessary to trade between the number of segments and the length on each segment in order to find an acceptable compromise between the bias error and the random error due to leakage noise and the random errors due to measurement noise in the estimated transfer function.

The systematic (bias) error due to leakage has been studied in this work for two common estimators; $H_{1}$ and $H_{2}$. It is shown that the resulting biased FRF depends on what type of estimator is used. In practice, it can therefore be beneficial to combine the results from both estimators in order to find a better approximation of the system's actual FRF.

An effective technique has been proposed in this work, based on using a sum of real exponentials to describe the effect of the window, which makes it possible to determine the relative bias error at the resonance frequency for different window functions. The calculations were carried out for a rectangular window and a Hanning window, but the principle can be applied to other window functions as well. The derived formulas give a simple relationship between bias error, frequency resolution and 3dB-bandwidth for the resonance.

A simulation study was carried out and the results from the bias expressions were found to agree very well with empirical results. By replacing the unknown parameters with the estimated parameters, these formulas can be useful for quantifying the bias errors in the FRF analysis. Further studies on the random error due to leakage noise with different estimators are recommended. 


\section{Acknowledgment}

Financial support from the Faculty Board of Blekinge Institute of Technology is gratefully acknowledged.

\section{References}

[1] P. Welch, The use of fast fourier transform for the estimation of power spectra: A method based on time averaging over short, modified periodograms, Audio and Electroacoustics, IEEE Transactions on 15 (June 1967), 70-73.

[2] P.M. Broersen, On bias in transfer functions estimated with stochastic excitation, IEEE International Conference on Acoustics, Speech, and Signal Processing, 1994, pp. IV/49 -IV/52 vol. 4.

[3] P.M. Broersen, A comparison of transfer function estimators, IEEE Transactions on Instrumentation and Measurement 44(3) (1995), $657-661$.

[4] J. Douce and L. Balmer, Statistics of frequency-response estimates, Control Theory and Applications, IEE Proceedings D 137(5) (1990), 290-296.

[5] J. Schoukens, Y. Rolain and R. Pintelon, Analysis of windowing/leakage effects in frequency response function measurements, Automatica 42 (2006), 27-38.

[6] J. Antoni and J. Schoukens, A comprehensive study of the bias and variance of frequency-response-function measurements: Optimal window selection and overlapping strategies, Automatica 43 (2007), 1723-1736.

[7] J. Antoni and J. Schoukens, Optimal settings for measuring frequency response function with weighted overlapped segment averaging, IEEE Transactions on Instrumentation and Measurement 58 (2009) 1764-1769.

[8] J.S. Bendat and A.G. Piersol, Random data - analysis and measurement procedures, John Wiley Sons, Inc., second ed., 1986.

[9] A. Brandt, Noise and Vibration Analysis - Signal Analysis and Experimental Procedures, John Wiley Sons, Inc., first ed., 2011.

[10] H.M. Hayes, Statistical Digital Signal Processing and Modeling, John Wiley Sons, Inc., first ed., 1996.

[11] A. Papoulis, Signal Analysis, McGraw-Hill, Inc., first ed., 1997.

[12] J.W. Tedesco, G.W. McDougal and C.A. Ross, Structural dynamics - theory and applications. Addison Wesley Longman, first ed., 1999.

[13] P. Boersma, Accurate short-term analysis of the fundamental frequency and the harmonics-to-noise-ratio of a sampled sound, Institute of Phonetic Sciences, Proceedings 17, 1993.

[14] K. Ahlin, M. Magnevall and A. Josefsson, Simulation of forced response in linear and nonlinear mechanical systems using digital filters, in Proceedings of the International Conference on Noise and Vibration Engineering (ISMA), (Leuven, Belgium), 2006. 

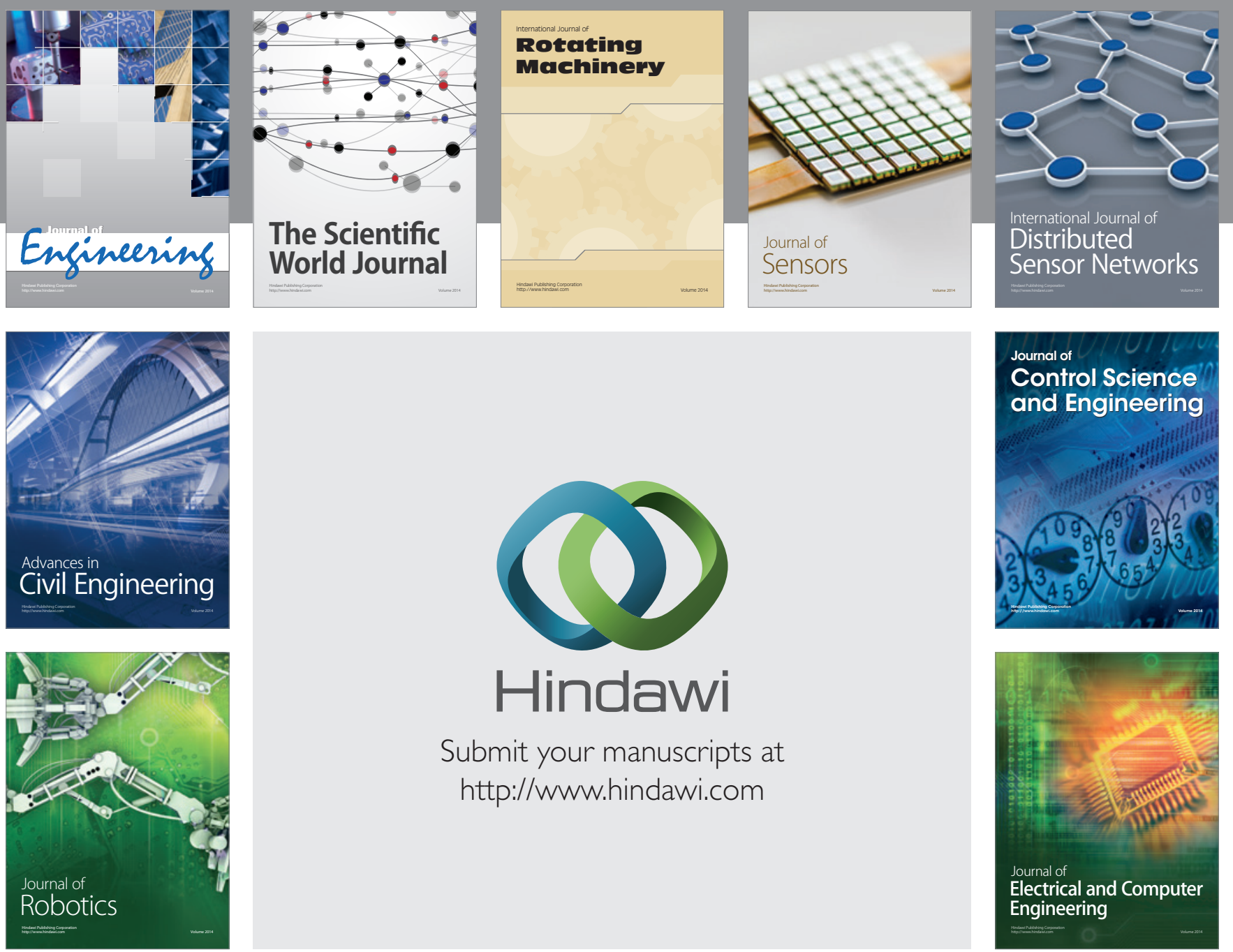

Submit your manuscripts at

http://www.hindawi.com
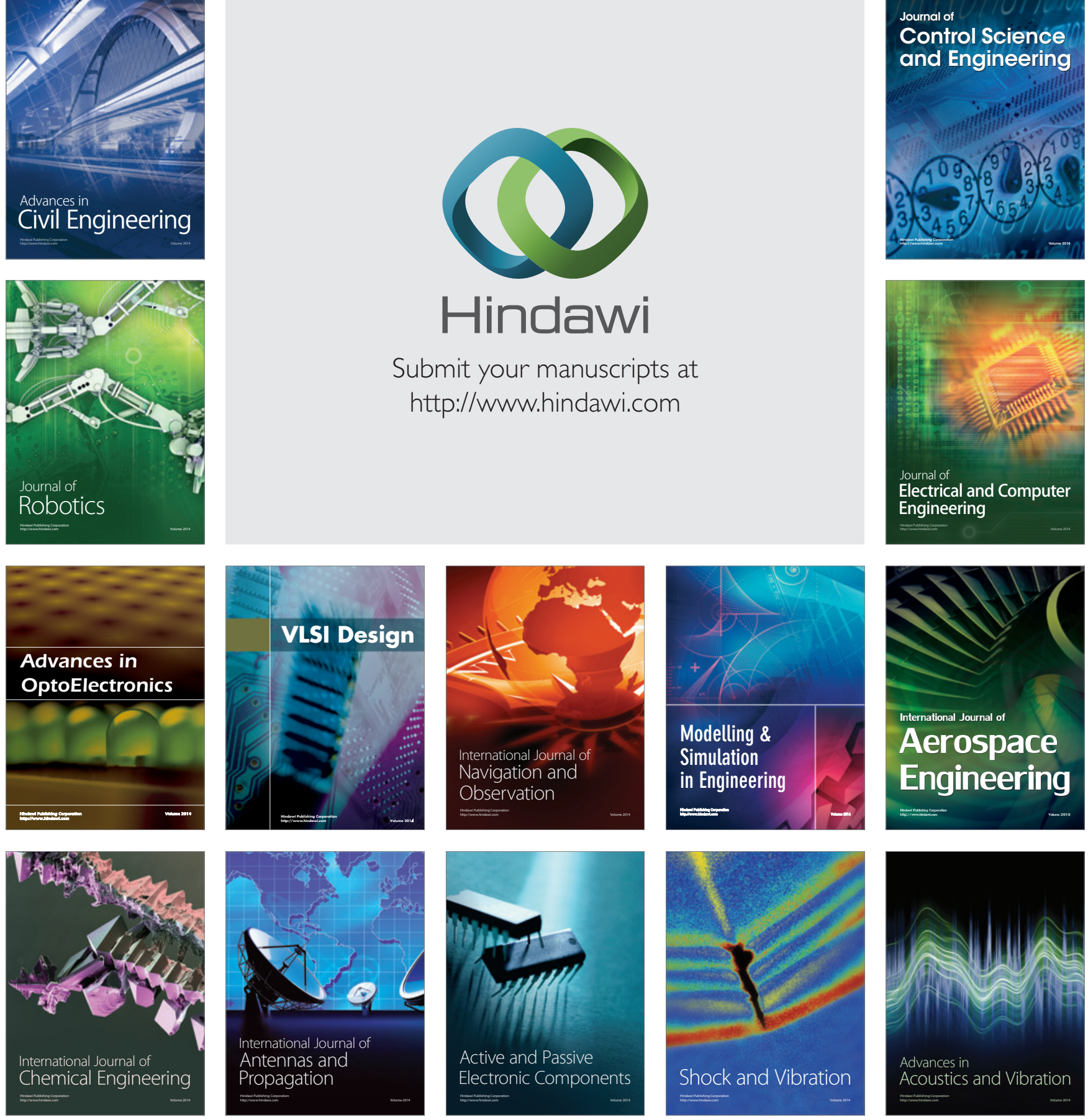